\title{
Human adipose-derived mesenchymal stem cells repair cisplatin-induced acute kidney injury through antiapoptotic pathways
}

\author{
WEIQI YAO ${ }^{1 *}$, QINYONG HU $^{2 *}$, YUHONG MA $^{1}$, WENPING XIONG ${ }^{1}$, \\ TINGTING WU ${ }^{1}, \mathrm{JUN} \mathrm{CAO}^{1}$ and DONGCHENG WU ${ }^{1}$ \\ ${ }^{1}$ Department of Biochemistry and Molecular Chemistry, School of Basic Medical Sciences; \\ ${ }^{2}$ Department of Oncology, Ren Ming Hospital, Wuhan University, Wuhan, Hubei 430071, P.R. China
}

Received April 24, 2014; Accepted January 5, 2015

DOI: $10.3892 /$ etm.2015.2505

\begin{abstract}
Cisplatin has been hypothesized to induce nephrotoxicity through triggering the apoptosis of tubular cells; however, the drug remains widely administered for the treatment of tumors. Recently, mesenchymal stem cells (MSCs) have been demonstrated to protect the kidney from the adverse effects induced by cisplatin. The aim of the present study was to investigate the mechanisms underlying the protective effects of human adipose-derived MSCs (AD-MSCs) on kidney function and tubular cells. Sprague-Dawley rats were divided into three groups, which included the healthy controls, those subjected to cisplatin-induced acute kidney injury (AKI) for $24 \mathrm{~h}$ without subsequent treatment and those subjected to cisplatin-induced AKI for $24 \mathrm{~h}$, followed by AD-MSC engraftment. The rats were sacrificed at day 5 and the effects were analyzed using various methods, including biochemical analysis, structural examination and cell tracking experiments. In addition, an in vitro experiment with NRK-52E cells was performed. The cells were divided into three groups, including the healthy control, cisplatin induction and cisplatin induction with co-culture of AD-MSCs, and were subsequently assessed with a Transwell assay. After culture for four days, the cells were lysed and the total protein extract was subjected to western blot analysis. Cisplatin-induced renal dysfunction and tissue damage was shown to recover following AD-MSC infusion, although there were few AD-MSCs observed around the injured kidney
\end{abstract}

Correspondence to: Professor Dongcheng $\mathrm{Wu}$, Department of Biochemistry and Molecular Chemistry, School of Basic Medical Sciences, Wuhan University, 185 Donghu Road, Wuhan, Hubei 430071, P.R. China

E-mail: ywyhcn@163.com

*Contributed equally

Key words: adipose derived-mesenchymal stem cells, acute kidney injury, antiapoptosis tubules in the kidney. When the cisplatin-treated NRK-52E cells were co-cultured with AD-MSCs, the activation of $\mathrm{p} 38$ and BAX were inhibited, while the expression of Bcl-2 was upregulated, as compared with the cisplatin-treated NRK-52E cells that were not co-cultured. Therefore, AD-MSCs were shown to markedly improve cisplatin-induced renal failure and tubular cells necrosis through the secretion of certain factors, which subsequently inhibited the apoptosis pathway in vitro. It was hypothesized that AD-MSC secretion was triggered by the injured tubular cells. Thus, AD-MSCs may be important for the therapy of patients with renal injury due to their antiapoptotic capacity.

\section{Introduction}

Cisplatin has been widely and effectively used for chemotherapy against a number of tumor types; however, the drug induces a series of side effects in various organs, of which the major organ affected is the kidney (1-3). Cisplatin triggers the apoptosis of renal tubular epithelial cells, which is followed by inflammation and fibrosis (4). These alterations predominantly occur in cells of the proximal tubule, particularly in the epithelial cells surrounding the S3 segment, since cisplatin accumulates at the highest concentrations in these areas (5). Previously, multiple mechanisms and signaling pathways associated with cisplatin-induced tubular cell death have been illustrated and considered as potential targets for clinical treatment (6-10).

The identification of a novel and effective treatment for cisplatin-induced renal injury has been prompted in this situation. Previous studies have demonstrated that mesenchymal stem cells (MSCs) have enormous potential to repair the injured tissues through infusion and paracrine signaling (11-13). MSCs are undifferentiated adult cells that can be isolated from a variety of tissues, but primarily from the bone marrow (BM). The cells emerge from mesenchymal cells that generate connective tissues, including bone, cartilage and fat, and blood supply-related organs, such as the vasculature and hematopoietic systems. MSCs are defined by their adherence to plastic in culture, multipotentiality (14) and their ability to be induced and differentiated into renal cells (15). Transplantation of 
BM-MSCs or other types of progenitor cells from rodents is a strategy for renal repair in experimental models of acute kidney injury (AKI) (16-21), a highly life-threatening clinical condition $(12,14,22)$. Currently, adipose-derived (AD)-MSCs have been increasingly recognized due to their similar capacity to BM-MSCs, exhibiting multi-differentiation and tissue repair properties. In addition, the collection of adipose tissue is easier since liposuction is widely used in clinical practice. Furthermore, it has been demonstrated that AD-MSCs are able to protect the kidney from renal failure (18,23-31).

The aim of the present study was to further investigate the functional effects of AD-MSCs on AKI. To the best of our knowledge, this is the first study to use human AD-MSCs for the treatment of animals with AKI. In addition, the current study applied detailed tracking following transplantation. The amelioration of renal function was evaluated via several aspects, including functional recovery at the biochemical level, reconstruction of the tubule structure with more proliferative epithelial cells, and the relevance between AD-MSC fusion and functional restoration of the kidney.

Furthermore, the present study utilized a co-culture system with two types of cell in a Transwell assay to analyze the antiapoptotic capacity of AD-MSCs in vitro. The antiapoptotic functions of AD-MSCs were analyzed with the aim to provide experimental evidence supporting the therapeutic potential for replacing MSCs in cell transplantation for human diseases.

\section{Materials and methods}

AD-MSC culture. AD-MSCs were isolated from human fat tissue. The adipose tissue was obtained from abdominal fat collected during liposuction surgery at Wuhan Mei Ji Yuan Plastic and Esthetic Surgery Hospital (Wuhan, China). The study was conducted in accordance with the Declaration of Helsinki, and with approval from the Ethics Committee of Wuhan University (Wuhan, China). Written informed consent was obtained from all the participants. Culture methods were based on a classical method and modified (32). Using liposuction, up to $20 \mathrm{ml}$ adipose tissue was obtained, which was centrifuged at $800 \times \mathrm{g}$ for $5 \mathrm{~min}$, after which the liquid was removed. The tissue samples were washed with phosphate-buffered saline (PBS; Sigma-Aldrich, St. Louis, MO, USA) three times, followed by digestion in $0.075 \%$ collagenase (Sigma-Aldrich), using the same volume as the tissue, for $30 \mathrm{~min}$ at $37^{\circ} \mathrm{C}$ whilst shaking. The sample was neutralized with Dulbecco's modified Eagle's medium (DMEM; Gibco Life Technologies, Carlsbad, CA, USA) containing 20\% fetal bovine serum (FBS; Gibco Life Technologies), and centrifuged at $1,000 \mathrm{x}$ g for $15 \mathrm{~min}$. The pellet was resuspended in PBS and passed through a $70-\mu \mathrm{m}$ cell strainer. The cells were cultured in low glucose-DMEM, supplemented with 20\% FBS and antibiotics. After $24 \mathrm{~h}$, the media were removed, and refreshed every three days until the cells reached $>70 \%$ confluence, after which the cells were passaged.

Phenotypic analysis and in vitro differentiation assays. AD-MSC phenotypic analysis was conducted using Cytomics FC500 (Beckman Coulter, Brea, CA, USA). The cells were harvested in passage three, washed in flow cytom- etry buffer and incubated for $20 \mathrm{~min}$ in flow cytometry buffer containing fluorescein-conjugated monoclonal antibodies directed against MSC antigens: phycoerythrin(PE)-conjugated anti-human CD13 (cat. no. 301704), fluorescein isothiocyanate (FITC)-conjugated anti-human CD90 (cat. no. 328108), allophycocyanin-conjugated anti-human CD44 (cat. no. 338806) and FITC-conjugated anti-human CD105 (cat. no. 323203), and against hematopoietic antigens: APC-conjugated anti-human CD14 (cat. no. 301808), PE-conjugated anti-human CD34 (cat. no. 343606), PE/Cy5-conjugated anti-human CD45 (cat. no. 304010) and FITC-conjugated anti-human CD71 (cat. no. 334104). All antibodies were purchased from BioLegend (San Diego, CA, USA).

AD-MSCs were obtained by plastic adhesion. As aforementioned, AD-MSCs have a similar differentiation capacity to MSCs derived from bone marrow. To confirm their pluripotency potential, AD-MSCs were cultured in induction media, which caused the cells to differentiate into osteoblasts, adipocytes and chondroblasts [osteoblasts (cat. no. HUXMD-90021), adipocytes (cat. no. HUXMA-90031) and chondroblasts (cat. no. HUXMA-90041/90042) differentiation kits; Cyagen Biosciences, Inc., Guangzhou, China).

Labeling the AD-MSCs and transplantation following AKI in vivo. The ability of the AD-MSCs to repair the kidney following injury and the underlying mechanisms associated with cell localization after transplantation were analyzed. AD-MSCs were labeled using a PKH-26 Red Fluorescence Cell Linker kit (Sigma-Aldrich), which is normally used for general cell membrane labeling according to Morigi et al (33). Next, the cells were transplanted into the rats who had been subjected to cisplatin-induced AKI. To confirm the cell viability when labeled with $\mathrm{PKH}-26$ and the dyeing efficiency, a 3-(4,5-dimethylthiazol-2-yl)-2,5-diphenyltetrazolium bromide (MTT) assay was performed, after which the stained cells were seeded on coverslips and observed under a microscope (SZ61GFP/TS; Olympus Corporation, Tokyo, Japan).

Male Sprague-Dawley rats (weight, 200-230 g) were provided with a standard rat chow and free access to water in a temperature- and humidity-controlled facility. The rats were subjected to a 12-h light/dark cycle. The study was carried out in strict accordance with the recommendations in the Guide for the Care and Use of Laboratory Animals of the National Institutes of Health. The animal use protocol was reviewed and approved by the Institutional Animal Care and Use Committee of Wuhan University. The rats were divided into the following three groups. Group 1 received saline injections and were referred to as the healthy control group $(n=10)$. Group 2 was the AD-MSC treatment group $(n=15)$, in which each rat was administered AD-MSCs $\left(1-2 \times 10^{6}\right.$ cells/1 ml saline) one day after cisplatin induction. Only the cells cultured in passage three were used for grafting. Finally, group 3 was the cisplatin injection group $(n=12)$ that were subjected to cisplatin-induced AKI, as with the rats in group 2. However, the rats in group 3 received a saline injection instead of the AD-MSCs. In all the groups, the cells and reagents were injected via the tail vein, with the exception of cisplatin and the first saline injection in group 1, where the reagents were injected into the peritoneal cavity. The rats in groups 2 and 3 were injected with cisplatin (Sigma-Aldrich) 
at a concentration of $6 \mathrm{mg} / \mathrm{kg}$ body weight. After five days, the rats were sacrificed by severing the carotid artery, and the kidneys were harvested. Tissues samples were sectioned for histology, apoptosis and proliferation and morphometric analyses. Since the AD-MSCs were obtained from human adipose tissue, the kidney sections of the animals injected with PKH-26-labeled cells were stained with a mouse anti-human monoclonal CD105 antibody (cat. no. MCA1557; AbD Serotec, Kidlington, UK), followed by an anti-mouse Cy5 antibody (Jackson ImmunoResearch Laboratories, Inc., West Grove, PA, USA). Five rats from group 2 were injected with AD-MSCs that had not been labeled with PKH-26, and the remaining 10 rats in group 2 were injected with AD-MSCs labeled with PKH-26; however, these sections were stained with Cy5-anti-human CD105 to detect the location of the AD-MSCs in the kidney. Samples were counterstained with FITC-labeled lectin wheat germ agglutinin (WGA; Vector Laboratories Ltd., Peterborough, UK), while the nuclei were stained with 4',6-diamidino-2-phenylindole dihydrochloride hydrate (DAPI; Sigma-Aldrich).

Determination of renal function. All the animals were sacrificed at day five following cisplatin induction and whole blood samples were collected. Rats were housed in metabolism cages (Nanjing Bian Zhen Bio-science Company, Nanjing, China), and the blood serum and urine were collected every $24 \mathrm{~h}$ for determination of renal function by biochemistry analysis, which included measurements of the serum blood urea nitrogen (BUN) level (48T/96T; Biofine, Blaine, WA, USA), the creatinine clearance rate (Ccr) (48T/96T; Shanghai Yanjin Bio-science Company, Shanghai, China), urinary microalbumin (mALB) (cat. no. ARB12655; Beijing Ai Ran Bio-tech Ltd., Beijing, China) and $\beta 2$ microglobulin $(\beta 2 \mathrm{mG})$ (Shanghai Yuanyan Bio-science Limited Company, Shanghai, China). These parameters were measured using standard diagnostic kits with a Clinical Chemistry Analyzer (AU400; Olympus Corporation).

Renal histology. After the animals were sacrificed, one side of the kidney tissues were removed by surgery and fixed in formalin. The tissues were sectioned into $5-\mu \mathrm{m}$ slices for paraffin embedding, which was followed by staining with hematoxylin and eosin (H\&E) or periodic acid-Schiff (PAS) reagent. Tubular injury was scored by grading the percentage of the affected tubules under ten randomly selected non-overlapping fields (magnification, $\mathrm{x} 200$ ). The scoring was conducted as follows: $0,0 \% ; 1, \leq 10 \% ; 2,11-25 \% ; 3,26-45 \% ; 4$, 46-75\%; and 5, 76-100\%. To score the injured tubules, whole tubular numbers per field were considered as standard under x200 magnification. Thus, the injury was calculated as follows: Injury score $(\%)=$ (number of injured tubules/number of whole tubules) x 100 (23).

A terminal transferase-mediated dUTP nick-end labeling (TUNEL) assay (DeadEnd ${ }^{\mathrm{TM}}$ Fluorometric TUNEL system; cat no. G3250; Promega Corporation, Madison, WI, USA) was used to identify the extent of apoptosis. Furthermore, the number of cells undergoing proliferation was measured through staining with a monoclonal anti-proliferating cell nuclear antigen (PCNA) antibody (cat. no. MA1-16827; Thermo Fisher Scientific, Waltham, MA, USA). Paraffin-embedded sections were stained with TUNEL and PCNA and observed under a microscope (LSM 700; Carl Zeiss AG, Oberkochen, Germany) at x400 magnification (34). In total, 20 areas were randomly selected for each slide to determine the result.

The other side of the kidney tissues were embedded in Tissue-Tek OCT Compound (cat. no. 4583; Sakura Finetek Japan Co., Ltd., Tokyo, Japan) and fresh-frozen in liquid nitrogen. The samples were sectioned into $8-\mu \mathrm{m}$ slices and stored at $-80^{\circ} \mathrm{C}$. The sections were subsequently fixed in acetone for $10 \mathrm{~min}$ and incubated with FITC-labeled lectin WGA, which binds membrane glycoproteins and sialic acid and was used for identifying the tubular structures. Nuclei were stained with DAPI. In total, 20 sections per mouse were analyzed.

Co-culture with AD-MSCs and injured NRK-52E cells. A specific rat renal proximal tubular cell line, NRK-52E (American Type Culture Collection, Manassas, VA, USA), was used to set up the co-culture model. This cell line had been previously used as in vitro model (34). The cells were cultivated according to recommended conditions, comprising DMEM supplemented with $10 \%$ heat-inactivated FBS, 2 mM glutamine (Gibco Life Technologies) and penicillin/streptomycin (100 U/ml; Gibco Life Technologies).

Cells were divided to three groups and cultured in six-well dishes. Group 1 NRK-52E cells were cultured without any treatment, while group 2 NRK-52E cells were induced by cisplatin. Group 3 NRK-52E cells were treated with cisplatin, in the same conditions as group 2, but were also co-cultured with AD-MSCs in a Transwell assay (pore size, $0.4 \mu \mathrm{m}$; Costar $^{\circledR}$; Corning Life Science, Corning, NY, USA). Cisplatin (100 mM) was added to groups 2 and 3 at a volume of $170 \mu \mathrm{l}$ over a period of $6 \mathrm{~h}$. In addition, for group 3, AD-MSCs were cultivated in Transwell chambers at a concentration of $2.5 \times 10^{5} / \mathrm{ml}$ in $2 \mathrm{ml}$ media for four days. NRK-52E cells were lysed after co-culture and the protein was extracted for western blot analysis. The expression levels of phosphorylated (p)-p38, B-cell lymphoma 2 (Bcl-2) and $\mathrm{Bcl}-2$-associated $\mathrm{X}$ protein (BAX) were analyzed using a standard protocol, which was used to identify the antiapoptotic mechanisms of AD-MSCs (34). Briefly, the cells were harvested and lysed in radioimmunoprecipitation assay buffer (Sigma-Aldrich). Protein concentrations of the lysates were quantified using Bicinchoninic Acid Protein Assay kit (Pierce Biotechnology, Inc., Rockford, IL, USA). Cell lysates were then loaded onto an $12 \%$ SDS-PAGE gel and separated by electrophoresis. Following electrophoresis, the proteins were transferred to polyvinylidene fluoride membranes (Bio-Rad Laboratories, Inc., Hercules, CA, USA) and non-specific binding sites were blocked with 5\% nonfat milk in PBS with $0.1 \%$ Tween-20. The membranes were then incubated with the following primary antibodies: Rabbit polyclonal anti-p-p38 (cat. no. ab4822; Abcam, Cabridge, MA, USA), rabbit polyclonal anti-BAX (cat. no. ab7977; Abcam); rabbit monoclonal anti-Bcl-2A1 (cat.no. ab33862; Abcam) and mouse monoclonal anti-actin (cat. no. A3853; Sigma-Aldrich) at $4^{\circ} \mathrm{C}$ overnight. The membranes were then incubated with HRP-conjugated secondary antibodies (Dako, Glostrup, Denmark) for $1 \mathrm{~h}$ at $4^{\circ} \mathrm{C}$, and visualized by enhanced chemiluminescence (GE Healthcare Life Sciences, Little Chalfont, UK). The blots were analyzed by ImageJ 1.48 software (National Institutes of Health, Bethesda, MD, USA). 

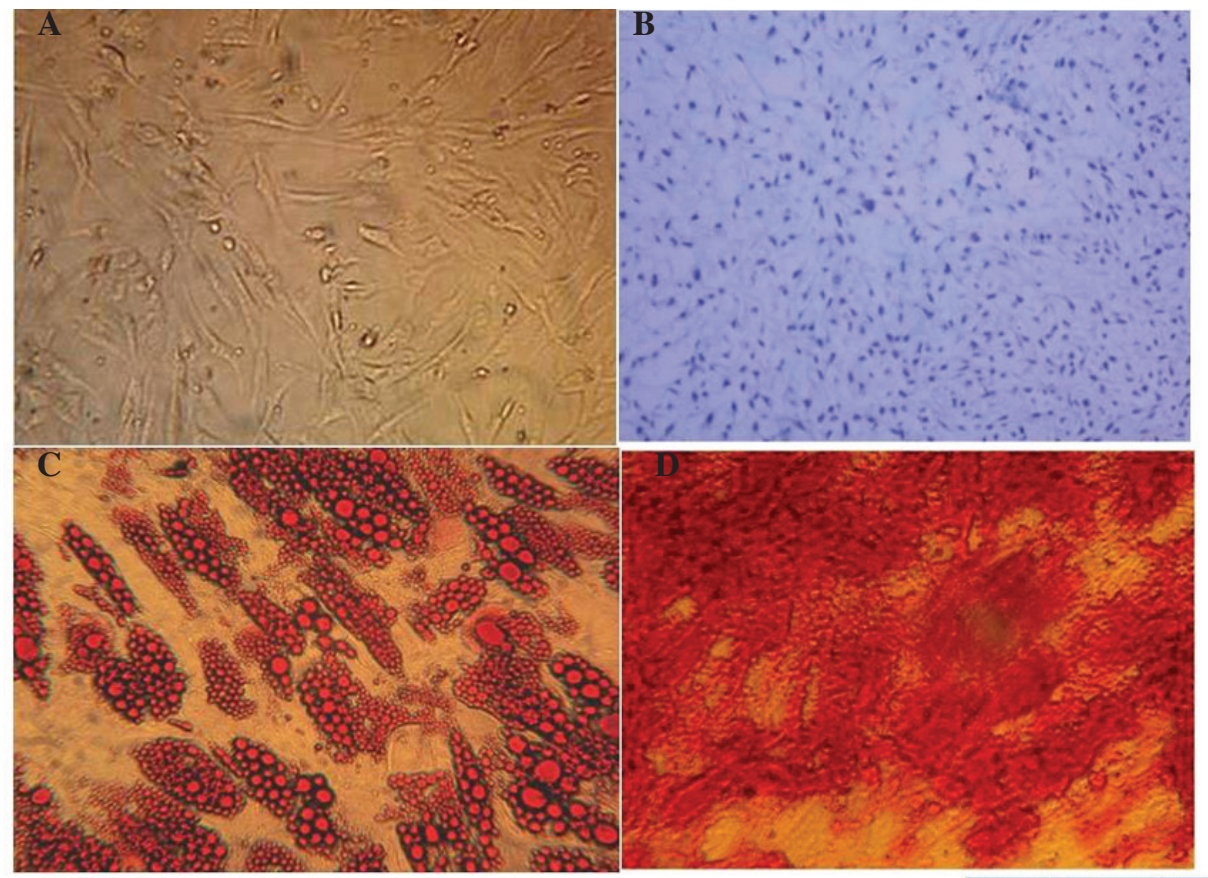

$\mathbf{E}$
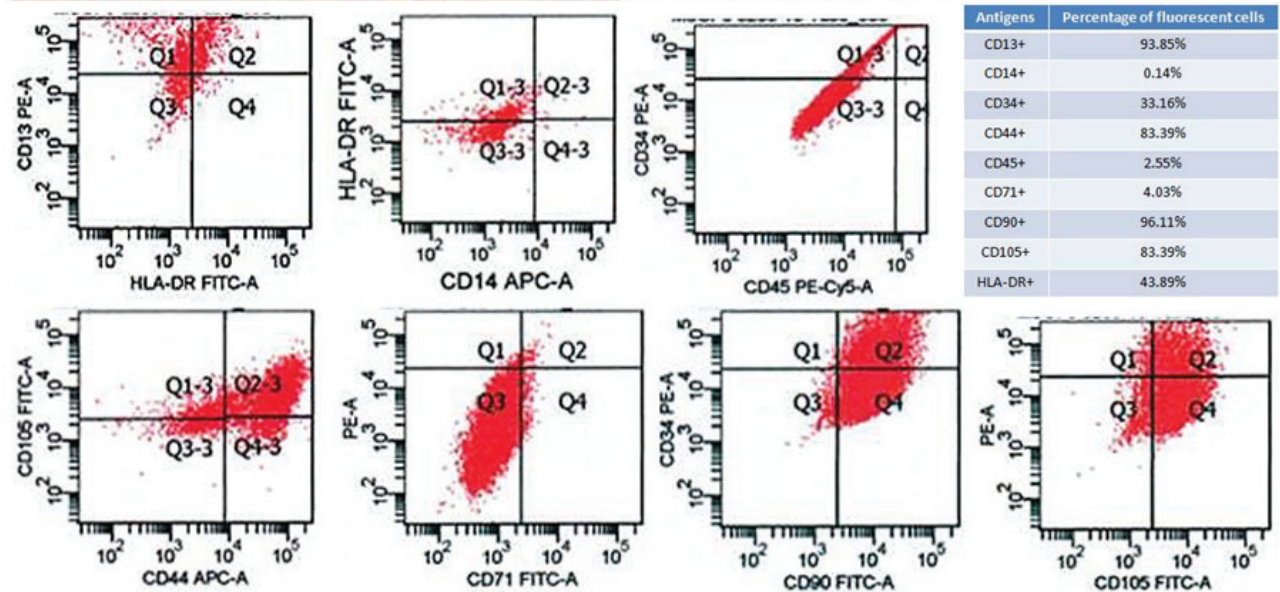

Figure 1. Characterization of human adipose-derived mesenchymal stem cells (AD-MSCs). (A) A representative phase-contrast microscopy image of AD-MSCs showing typical spindle-shaped morphology (magnification, x100). (B) Differentiation of cartilage cells (magnification, x400). (C) Adipocyte differentiation (magnification, x400). (D) Osteogenic differentiation of AD-MSCs stained with Alizarin Red (magnification, x400). (E) Fluorescence-activated cell sorting characterization of AD-MSCs expressing CD13, CD90, CD44 and CD105, the commonly used surface markers for MSCs. MSCs were also shown to express CD14, CD34, CD45 and CD71 at a very low level. FITC, fluorescein isothiocyanate; PE, phycoerythrin.

Statistical analysis. Results are expressed as the mean \pm standard error of the mean. Data were collected, and analyzed using the t-test and one-way analysis of variance. Statistical analysis was performed using SPSS 13.0 software (SPSS, Inc., Chicago, IL, USA), and $\mathrm{P}<0.05$ was considered to indicate a statistically significant difference.

\section{Results}

Isolation and characterization of human AD-MSCs. Human AD-MSCs were isolated from human fat tissue that was obtained from abdominal fat during liposuction surgery. Cultured cells were adherent to the dish and were shown to be morphologically flattened and spindle-shaped under a light microscope (Fig. 1A). The pluripotency of the AD-MSCs was demonstrated with distinct culture conditions that enabled the cells to differentiate into osteoblasts, adipocytes and chondroblasts (Fig. 1B-D).
To further characterize the human AD-MSCs, fluorescence-activated cell sorting analysis was performed to confirm that the cells endogenously expressed CD13, CD90, CD44 and CD105, which are the most important surface markers for MSCs. However, other surface markers, including CD14, CD34, CD45 and CD71, were rarely expressed on these cells (Fig. 1E).

AD-MSC engraftment protects the kidney in vivo. To determine whether the AD-MSCs were able to ameliorate renal function and structure following AKI, serum and urine samples were collected from the experimental rats every day, from which the levels of BUN, Ccr, urinary mALB and $\beta 2 \mathrm{mG}$ were analyzed. The measurements were compared among the three groups of male rats, including the healthy controls, the rats treated with cisplatin and the rats grafted with AD-MSCs following cisplatin treatment. 
A
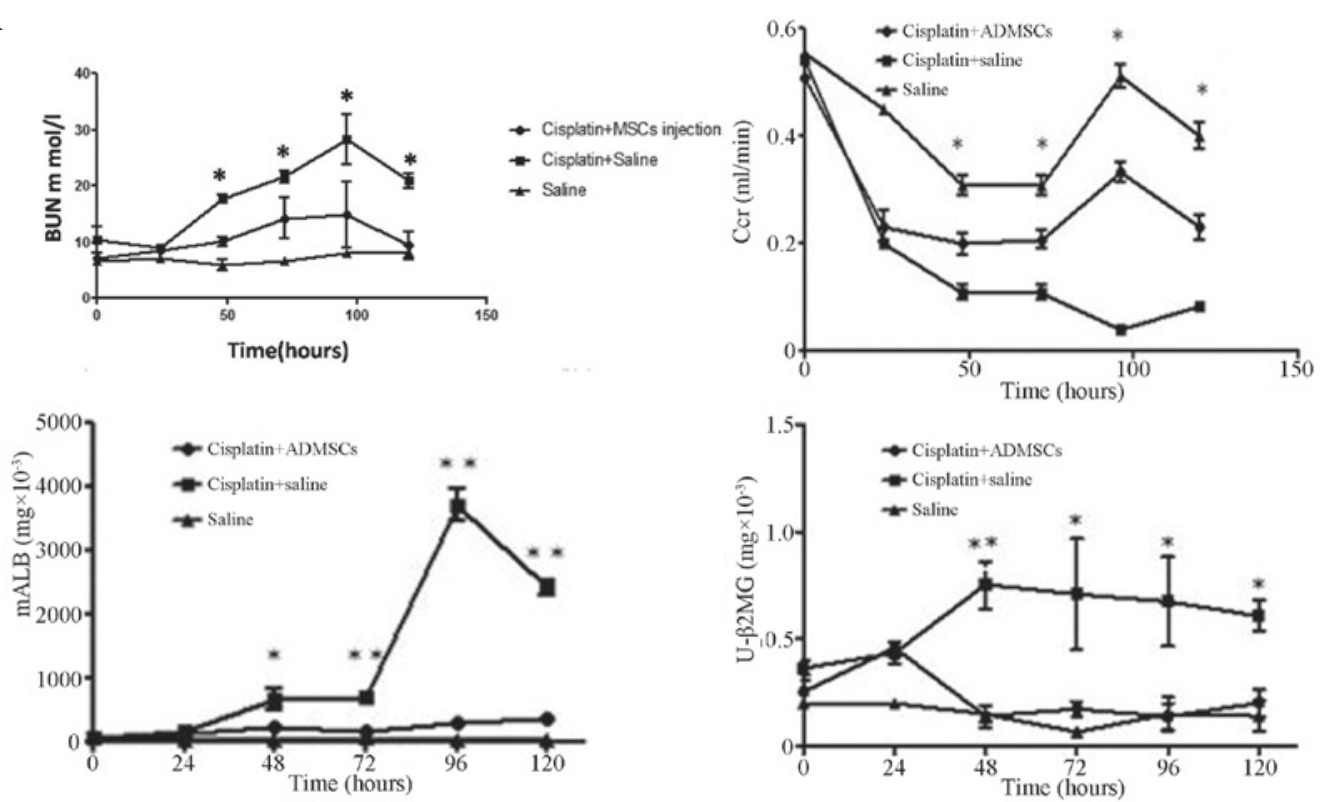

B

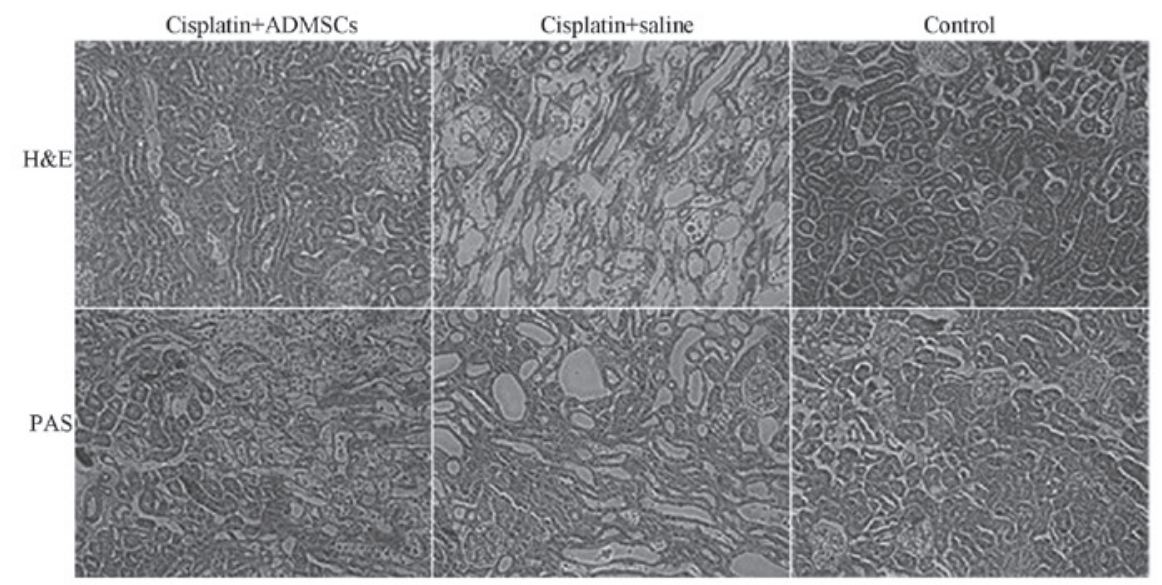

$\mathbf{C}$
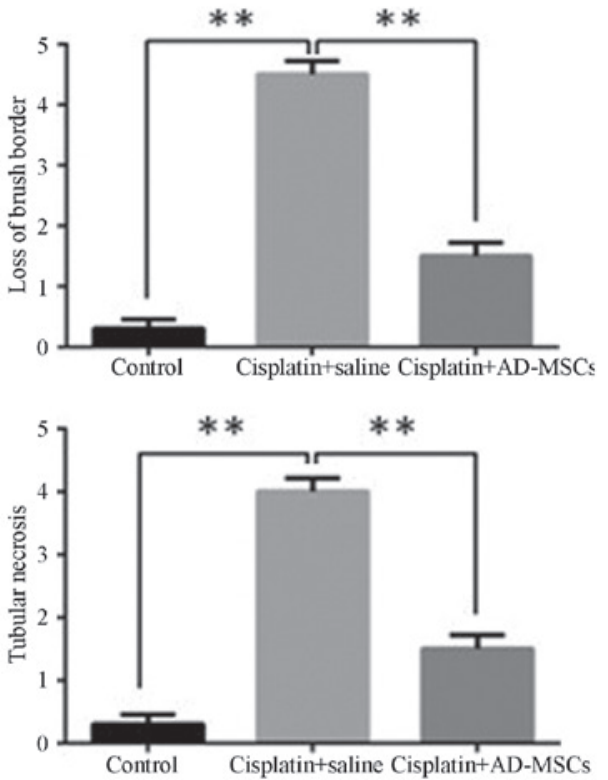
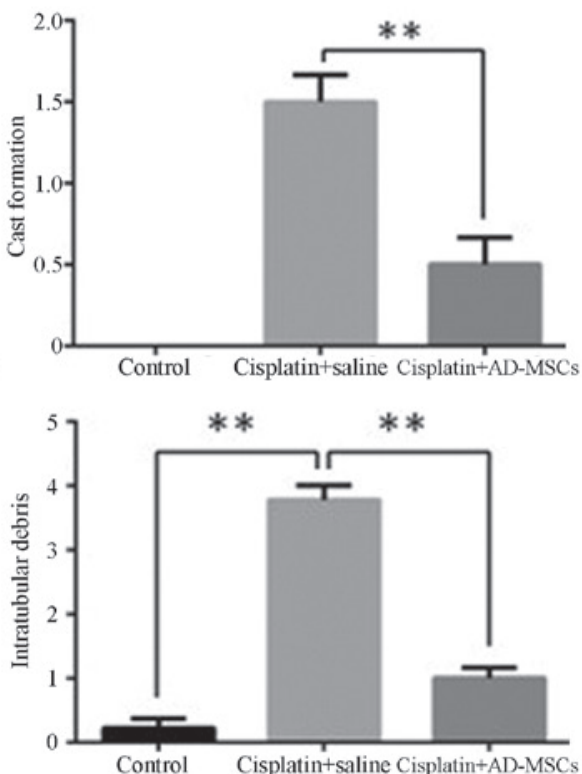

Figure 2. Kidney functional recovery and structural repair using human AD-MSCs in animals with acute kidney injury (AKI). (A) Renal function was assessed daily by the levels of BUN, serum Ccr, urinary mALB and $\beta 2 \mathrm{mG}$. Data are presented as mean \pm standard error of the mean $(\mathrm{SEM})$. ${ }^{*} \mathrm{P}<0.01$ and ${ }^{* *} \mathrm{P}<0.005$, cisplatin + saline group vs. cisplatin + AD-MSCs group. (B) Representative images from H\&E and PAS staining of the kidney sections from the healthy control, cisplatin + saline and cisplatin + human AD-MSCs groups (magnification, $\mathrm{x}$ 200). (C) Histopathological scoring of cisplatin-induced kidney tubular injury, as indicated with tubular necrosis, cast formation, loss of brush border and intratubular debris. Histopathological scoring was based on the percentage of affected tubules in the kidney sections. Data are presented as the mean \pm SEM. ${ }^{* *} \mathrm{P}<0.005$, between indicated groups. AD-MSCs, adipose-derived mesenchymal stem cells; BUN, blood urea nitrogen; Ccr, creatinine clearance rate; $\mathrm{mALB}$, microalbumin; $\beta 2 \mathrm{mG}$, microglobulin; U, urinary; H\&E, hematoxylin and eosin; PAS, periodic acid-Schiff. 
$\mathbf{A}$

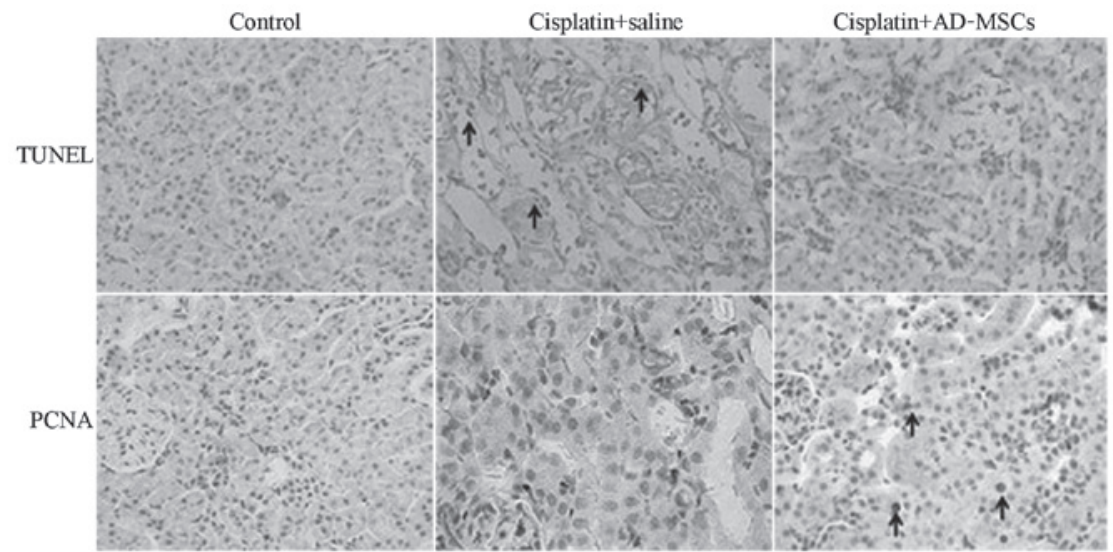

B

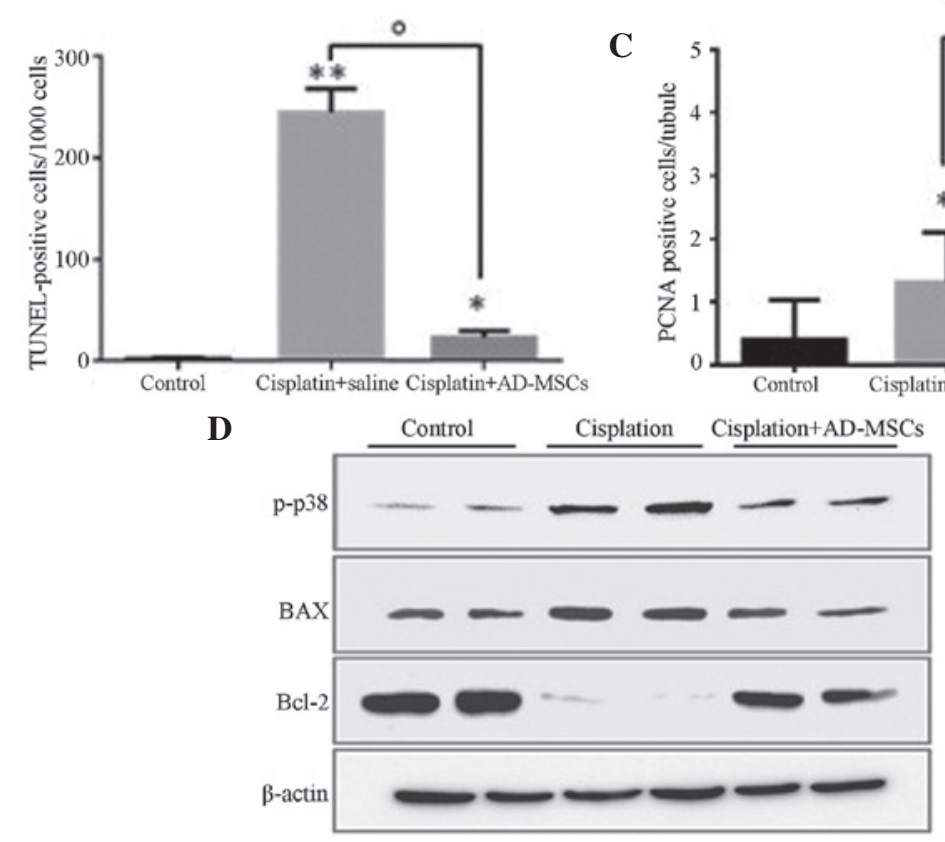

Figure 3. AD-MSCs significantly reduced the extent of apoptosis and promoted tubular cell proliferation in cisplatin-induced injured kidney tissues. (A) Representative images (magnification, $\mathrm{x} 400$ ) from TUNEL and PCNA staining in the kidney sections of the healthy control, cisplatin + saline and cisplatin + AD-MSCs groups. (B) Apoptotic cells and (C) PCNA-positive tubular cells were quantified for each kidney section. ${ }^{*} \mathrm{P}<0.01$ and ${ }^{* *} \mathrm{P}<0.005$, vs. control; ${ }^{\circ} \mathrm{P}<0.01$, between the indicated groups. Data are presented as the mean \pm standard error of the mean. (D) Immunoblot analysis examining the phosphorylation of p38 and the expression levels of the apoptotic regulators, BAX and Bcl-2. $\beta$-actin was used as a loading control. A representative blot from three independent experiments is shown. AD-MSCs, adipose-derived mesenchymal stem cells; TUNEL, terminal transferase-mediated dUTP nick-end labeling; PCNA, proliferating cell nuclear antigen, p-p38, phosphorylated p38.

The level of BUN markedly increased within the four-day period following the cisplatin injection, with the levels peaking between days 4 and 5 in the group of rats who were injected with cisplatin (11). For the rats treated with AD-MSCs, the level of BUN was significantly decreased compared with the non-treatment group $(\mathrm{P}<0.01)$. No statistically difference was observed in the BUN level between the AD-MSC-treated group and the healthy control group. Similarly, the Ccr in the AD-MSC-treated group was significantly reduced in contrast to the non-treatment group $(\mathrm{P}<0.01)$. Notably, the levels of $\mathrm{mALB}$ and $\beta 2 \mathrm{mG}$ in the AD-MSC-treated group were significantly decreased when compared with the non-treatment AKI group; however, the levels were similar to those observed in the healthy controls (Fig. 2A).

To evaluate the integrity of the renal structures, H\&E or PAS staining were performed on the kidney sections. The results clearly demonstrated that the injured kidneys were notably recovered when treated with the AD-MSCs, with observations morphologically similar to those of the healthy control group rats (Fig. 2B), Furthermore, a scoring system was introduced to quantify the injury of the kidney tubules. In the cisplatin-induced injury group, a loss of the brush border, flattening and a loss of epithelial cells were observed, as well as luminal cell debris and hyaline casts in the proximal tubules. However, when the rats were treated with AD-MSCs, the histopathological scores were significantly reduced compared with the injury group $(\mathrm{P}<0.005$; Fig. $2 \mathrm{C})$, which indicated engraftment of AD-MSCs effectively promoted the recovery of the tubular structure.

Human AD-MSC treatment reduces apoptosis and promotes cell proliferation. BM-MSCs have been shown to repair kidneys following AKI through inhibiting apoptosis and increasing DNA synthesis (unpublished data). Therefore, the present 

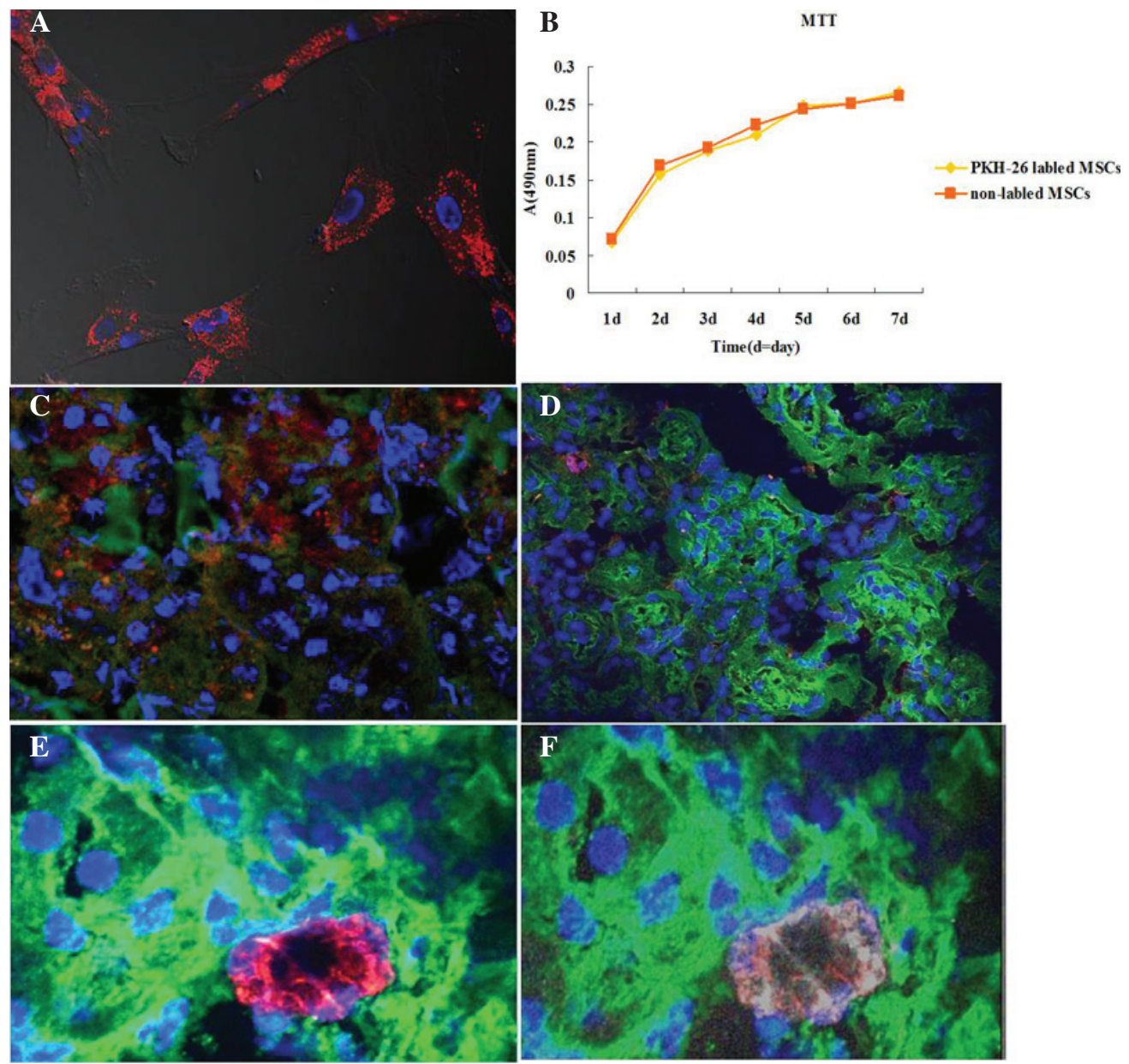

Figure 4. Tracking of human adipose-derived (AD)-MSCs through the injured kidneys induced with cisplatin (magnification, x630). (A) AD-MSCs were cultured on cover slips and stained with PKH-26 (red); nuclei were stained with 4',6-diamidino-2-phenylindole dihydrochloride hydrate (DAPI; blue). (B) A 3-(4,5-dimethylthiazol-2-yl)-2,5-diphenyltetrazolium bromide (MTT) assay indicated that PKH-26 labeling did not affect the viability and proliferation of AD-MSCs. (C) AD-MSCs-treated kidney sections were stained with fluorescein isothiocyanate (FITC)-labeled wheat germ agglutinin (WGA; green), PKH-26 (red) and DAPI (blue). As observed, PKH-26-labeled MSCs were located around the tubules. (D) Following an injection of AD-MSCs without PKH-26 labeling, the kidney sections were stained with Cy5-anti-human CD105 (red), FITC-labeled WGA and DAPI (blue). (E and F) PKH-26-positive cells (red) were localized around the tubules, and co-stained with Cy5-anti-CD105 (white), while nuclei were stained with DAPI (blue). MSCs, mesenchymal stem cells; A, absorbance.

study investigated whether AD-MSCs behave similarly in the injured kidneys. A TUNEL assay was performed, and the results revealed that the percentage of apoptotic cells (counted in every $1 \times 10^{3}$ cells) was highly reduced in the AD-MSC treatment group when compared with the cisplatin-induced injury group, exhibiting similar levels to those in the healthy control group (Fig. 3A and B).

PCNA staining was also performed to evaluate the proliferative capacity of the tubular cells in the injured kidneys treated with AD-MSCs. The results revealed that the number of PCNA-positive cells significantly increased in the AKI rats when compared with the control group $(\mathrm{P}<0.01)$, which indicated that a self-repair system may be stimulated in the injured kidneys (Fig. 3A and B). As expected, the number of proliferative cells/tubules labeled with PCNA in the AD-MSC-treated group was significantly higher compared with the cisplatin-induced group without treatment $(\mathrm{P}<0.01)$. These results indicated that AD-MSCs were able to repair the kidneys with AKI through inhibiting apoptosis and promoting tubular cell proliferation (Fig. 3A and B).
Tracking of human AD-MSCs in vivo following engraftment. To further understand the mechanisms underlying the effects of AD-MSCs in injured kidneys, AD-MSCs were tracked in vivo following engraftment. Prior to injection with PKH-26-labeled AD-MSCs into the injury-induced rats, the binding efficiency of PKH-26 and the effects on cell proliferation to the cultured AD-MSCs were investigated. The results revealed that PKH-26 was able to effectively label the AD-MSCs (Fig. 4A) and were experimentally feasible (Fig. 4B). Notably, only a small number of positive cells were found to be located around the kidney tubules in each section (red; Fig. 4C). However, there were a greater number of PKH-26 labeled cells located in the liver and spleen (data not shown). To further confirm the localization of the AD-MSCs in the kidney tubules, the human AD-MSCs were stained with the specific surface marker, CD-105 (human). Similarly, this marker was detected in a small number of cells around the tubules (red, Fig. 4D). To further verify the localization of the AD-MSCs in the injured kidneys, the AD-MSCs were co-stained with CD105 (mouse anti-human) and PKH-26, avoiding discrepancy due to 
membrane fluidity. PKH-26 and CD-105 double-positive cells were only identified in the cell infusion rat sections; however, they were not observed in all the fields (Fig. 4E and F). These results indicated that $\mathrm{AD}-\mathrm{MSC}$ were located around the tubules in the cortex, repairing the tubular cells; however, the number of cells was very small.

Treatment of human AD-MSCs in vitro. The $\mathrm{p} 38$ mitogen-activated protein kinase (MAPK) signaling pathway plays a significant role in apoptosis (35), and AD-MSCs have been shown to strongly inhibit the phosphorylation of p38 in cisplatin-induced AKI in vivo (23). Furthermore, Bcl-2 family members, who are crucial regulators of apoptosis, have been demonstrated to play key roles in AKI induced by cisplatin (36). However, whether the apoptotic function of MSCs, particularly AD-MSCs, results from cell fusion or the secretion of certain factors is yet to be determined. To investigate further, NRK-52E cells and AD-MSCs were co-cultured without direct contact, after which an immune-blot was performed. The levels of p-p38 and BAX were shown to be upregulated, while the expression levels of Bcl-2 were markedly downregulated in the NRK-52E cells cultured with the cisplatin-treated tubular cells, as compared with the cells from the healthy control. However, co-culture of AD-MSCs with NRK-52E cells was shown to eliminate the dysregulated effect of these proteins in NRK-52E cells (Fig. 3C). Therefore, these results indicated that the mechanisms underlying the modulation of the apoptotic pathways by AD-MSCs in the repair of injured tubular cells involved the release of a substance into the media, which was produced following the stimulation of the injured cells.

\section{Discussion}

The roles of human BM-MSCs in AKI repair have been well documented $(11,12,14,33,34)$. However, as a new potential therapeutic source, AD-MSCs may be able to replace BM-MSCs due to their easier method of collection, improved proliferative capacity and easier induction into the epithelium (24,25).

The function of injured kidneys has been shown to recover well following MSC engraftment, as indicated by BUN levels, Ccr values and observations of tubular structures $(14,18,23,30)$. In the present study, the results from a serial analysis of biochemical indicators, including BUN, Ccr, mALB and $\beta 2 \mathrm{mG}$, demonstrated that human AD-MSCs were able to largely ameliorate kidney function in animals with AKI. In addition, the observations from H\&E and PAS staining revealed that a number of glomeruli were damaged, which indicated that the rats treated with cisplatin may suffer kidney injury at a later stage. However, damaged glomerluli were not observed in the AD-MSC group, indicating that AD-MSCs were able to ameliorate AKI at an early developmental stage of renal failure, preventing glomeruli damage at the later stage.

AD-MSCs were tracked using PKH-26 and CD105. Notably, only a small number of positive cells were detected around the kidney tubules, with a greater number of cells visualized in other organs, including the liver and spleen (data not shown). These results indicated that AD-MSCs protect the kidneys against nephrotoxicity primarily through the release of certain types of factors, rather than the fusion of the cells to the injured tubules, which is similar to the behaviors of BM-MSCs and cord blood MSCs $(12,33)$. In addition, it was hypothesized that the secreted factors may reach the injured tubules through the blood circulation, instead of the paracrine system, as previously demonstrated (unpublished data). This hypothesis was formed since during paracrine signaling, the cells produce signals, including cytokines, which induce changes to neighboring cells (including damaged cells); however, only a small number of AD-MSCs were identified around the damaged tubular cells in the kidney sections.

Apoptosis and necrosis of tubular cells have been considered as the major consequences of cisplatin-induced AKI $(6,10,36)$. Human AD-MSCs have been shown to exhibit antiapoptotic capacity in vivo $(23,28)$. In the present study, the results from the TUNEL assay further confirmed the antiapoptotic function of AD-MSCs. In addition, p38 plays a critical role in cisplatin-induced kidney injury. The function of injured kidneys has been reported to improve following inhibition of the p38 signaling pathway (35).

In the present study, a co-culture system of AD-MSCs and cisplatin-induced human tubular cells was developed to investigate the antiapoptotic effects of human AD-MSCs. The results from western blot analysis demonstrated that human tubule cell injury may have triggered the AD-MSCs to secrete multiple factors that inhibit p38 activation, without the occurrence of cell fusion. BAX and Bcl-2 are two key regulators (proapoptotic and antiapoptotic, respectively) of apoptosis. Previous studies have demonstrated that MSC engraftment downregulates the expression level of BAX, but upregulates the expression level of $\mathrm{Bcl}-2$ in kidneys with AKI, which modulates their expression back to a control level $(13,23,36)$. In the current in vitro co-culture system, the expression patterns of three key regulators (p38, $\mathrm{BAX}$ and $\mathrm{Bcl}-2$ ) of apoptosis were clearly demonstrated following AD-MSC treatment, which was an important addition to the in vivo data demonstrating that AD-MSCs are able to effectively regulate apoptotic proteins (23). More importantly, these in vitro results strongly indicate that AD-MSCs are able to inhibit the apoptosis of NRK-52E cells using a mechanism of producing certain cytokines that are diffused in the media to reach NRK-52E cells, since this system was completely independent of cell-cell contact and cell fusion.

In conclusion, the present study demonstrated that AD-MSCs were able to repair cisplatin-induced AKI in rodents, as evidenced by biochemical indicators and observations of the kidney structures. According to the data from the co-culture experiments, a possible mechanism of kidney recovery with AD-MSC treatment may be that certain signals are released from the injured tubular cells that trigger AD-MSCs to produce specific cytokines. These are able to inhibit p38 MAPK activation, which results in the modulation of the expression levels of BAX and Bcl-2 to the level of a healthy control. As shown by the in vivo data, only a small number of AD-MSCs were identified around the injured tubules following engraftment. Therefore, from the in vitro and in vivo data, it was hypothesized that the cytokines produced by AD-MSCs reach the injured renal tubules through the blood circulation, not through the paracrine system, as described in a previous study (23). The results of the present study indicate that the cytokines produced by AD-MSCs are potentially able to replace cell therapy, which may resolve the safety issues from cell transplantation. 


\section{References}

1. Lebwohl D and Canetta R: Clinical development of platinum complexes in cancer therapy: an historical perspective and an update. Eur J Cancer 34: 1522-1534, 1998.

2. dos Santos NA, Carvalho Rodrigues MA, Martins NM and dos Santos AC: Cisplatin-induced nephrotoxicity and targets of nephroprotection: an update. Arch Toxicol 86: 1233-1250, 2012

3. Heidemann HT, Müller S, Mertins L, Stepan G, Hoffmann K and Ohnhaus EE: Effect of aminophylline on cisplatin nephrotoxicity in the rat. Br J Pharmacol 97: 313-318, 1989.

4. Francescato HD, Costa RS, Scavone C and Coimbra TM: Parthenolide reduces cisplatin-induced renal damage. Toxicology 230: 64-75, 2007.

5. Werner M, Costa MJ, Mitchell LG and Nayar R: Nephrotoxicity of xenobiotics. Clin Chim Acta 237: 107-154, 1995.

6. Jiang $\mathrm{M}$ and Dong Z: Regulation and pathological role of $\mathrm{p} 53$ in cisplatin nephrotoxicity. J Pharmacol Exp Ther 327: 300-307, 2008.

7. Clark JS, Faisal A, Baliga R, Nagamine Y and Arany I: Cisplatin induces apoptosis through the ERK-p66shc pathway in renal proximal tubule cells. Cancer Lett 297: 165-170, 2010.

8. Ramesh G and Reeves WB: TNF-alpha mediates chemokine and cytokine expression and renal injury in cisplatin nephrotoxicity. J Clin Invest 110: 835-842, 2002.

9. Zhang B,RameshG,Norbury CC and Reeves WB: Cisplatin-induced nephrotoxicity is mediated by tumor necrosis factor-alpha produced by renal parenchymal cells. Kidney Int 72: 37-44, 2007.

10. Zhuang S and Schnellmann RG: A death-promoting role for extracellular signal-regulated kinase. J Pharmacol Exp Ther 319: 991-997, 2006

11. Morigi M, Imberti B, Zoja C, et al: Mesenchymal stem cells are renotropic, helping to repair the kidney and improve function in acute renal failure. J Am Soc Nephrol 15: 1794-1804, 2004

12. Morigi M, Rota C, Montemurro T, et al: Life-sparing effect of human cord blood-mesenchymal stem cells in experimental acute kidney injury. Stem Cells 28: 513-522, 2010.

13. Peng X, Xu H, Zhou Y, et al: Human umbilical cord mesenchymal stem cells attenuate cisplatin-induced acute and chronic renal injury. Exp Biol Med (Maywood) 238: 960-970, 2013

14. Humphreys BD and Bonventre JV: Mesenchymal stem cells in acute kidney injury. Annu Rev Med 59: 311-325, 2008.

15. Kusaba T, Lalli M,Kramann R, Kobayashi A and Humphreys BD: Differentiated kidney epithelial cells repair injured proximal tubule. Proc Natl Acad Sci USA 111: 1527-1532, 2014

16. Maeshima A, Yamashita S and Nojima Y: Identification of renal progenitor-like tubular cells that participate in the regeneration processes of the kidney. J Am Soc Nephrol 14: 3138-3146, 2003.

17. Tadagavadi RK and Reeves WB: Renal dendritic cells ameliorate nephrotoxic acute kidney injury. J Am Soc Nephrol 21: 53-63, 2010

18. Zhu XY, Urbieta-Caceres V, Krier JD, Textor SC, Lerman A and Lerman LO: Mesenchymal stem cells and endothelial progenitor cells decrease renal injury in experimental swine renal artery stenosis through different mechanisms. Stem Cells 31: 117-125, 2013.

19. Altun B, Yilmaz R, Aki T, et al: Use of mesenchymal stem cells and darbepoetin improve ischemia-induced acute kidney injury outcomes. Am J Nephrol 35: 531-539, 2012.
20. Bian X, Zhang B, Guo W, et al: Effects of mesenchymal stem cells transplanted at different time points in a rat remnant kidney model. Am J Nephrol 39: 75-84, 2014.

21. Bussolati B, Tetta C and Camussi G: Contribution of stem cells to kidney repair. Am J Nephrol 28: 813-822, 2008.

22. Kitamura S, Yamasaki Y, Kinomura M, Sugaya T, Sugiyama H, Maeshima Y and Makino H: Establishment and characterization of renal progenitor like cells from S3 segment of nephron in rat adult kidney. FASEB J 19: 1789-1797, 2005.

23. Kim JH, Park DJ, Yun JC, et al: Human adipose tissue-derived mesenchymal stem cells protect kidneys from cisplatin nephrotoxicity in rats. Am J Physiol Renal Physiol 302: F1141-F1150, 2012.

24. Reinders ME and Rabelink TJ: Adipose tissue-derived stem cells: can impure cell preparations give pure results? Nephrol Dial Transplant 25: 3805-3807, 2010.

25. Brzoska M, Geiger H, Gauer S and Baer P: Epithelial differentiation of human adipose tissue-derived adult stem cells. Biochem Biophys Res Commun 330: 142-150, 2005.

26. Huang HC, Chang YJ, Chen WC, Harn HI, Tang MJ and Wu CC: Enhancement of renal epithelial cell functions through microfluidic-based coculture with adipose-derived stem cells. Tissue Eng Part A 19: 2024-2034, 2013.

27. Katsuno T, Ozaki T, Saka Y, et al: Low serum cultured adipose tissue-derived stromal cells ameliorate acute kidney injury in rats. Cell Transplant 22: 287-297, 2013.

28. Shih YC, Lee PY, Cheng H, Tsai CH, Ma H and Tarng DC: Adipose-derived stem cells exhibit antioxidative and antiapoptotic properties to rescue ischemic acute kidney injury in rats. Plast Reconstr Surg 132: 940e-951e, 2013.

29. Shin S, Kim Y, Jeong S, Hong S, Kim I, Lee W and Choi S: The therapeutic effect of human adult stem cells derived from adipose tissue in endotoxemic rat model. Int J Med Sci 10: 8-18, 2013

30. Wang W, Wang W, Jiang Y, Li Z, et al: Human adipose-derived stem cells modified by HIF-1 $\alpha$ accelerate the recovery of cisplatin-induced acute renal injury in vitro. Biotechnol Lett 36: 667-676, 2014

31. Zhang L, Li K, Liu X, et al: Repeated systemic administration of human adipose-derived stem cells attenuates overt diabetic nephropathy in rats. Stem Cells Dev 22: 3074-3086, 2013

32. Zuk PA,Zhu M, Ashjian P, et al: Human adipose tissue is a source of multipotent stem cells. Mol Biol Cell 13: 4279-4295, 2002.

33. Morigi M, Introna M, Imberti B, et al: Human bone marrow mesenchymal stem cells accelerate recovery of acute renal injury and prolong survival in mice. Stem Cells 26: 2075-2082, 2008.

34. Qi S and Wu D: Bone marrow-derived mesenchymal stem cells protect against cisplatin-induced acute kidney injury in rats by inhibiting cell apoptosis. Int J Mol Med 32: 1262-1272, 2013.

35. Francescato HD, Costa RS, da Silva CG and Coimbra TM: Treatment with a p38 MAPK inhibitor attenuates cisplatin nephrotoxicity starting after the beginning of renal damage. Life Sci 84: 590-597, 2009.

36. Iwayama $\mathrm{H}$ and Ueda $\mathrm{N}$ : Role of mitochondrial Bax, caspases, and MAPKs for ceramide-induced apoptosis in renal proximal tubular cells. Mol Cell Biochem 379: 37-42, 2013. 